http://dx.doi.org/10.35381/r.k.v6i2.1235

\title{
Práctica de la halterofilia a edades tempranas. Consecuencias en el crecimiento
}

\section{Practice of halterophilia at early ages. Consequences in the growth}

\author{
Jonattan Felipe Durán-Jácome \\ jonattan.duran@est.ucacue.edu.ec \\ Universidad Católica de Cuenca, Azogues \\ Ecuador \\ https://orcid.org/0000-0002-1950-4458 \\ Zoila Torres-Palchisaca \\ ztorresp@ucacue.edu.ec \\ Universidad Católica de Cuenca, Cuenca \\ Ecuador \\ https://orcid.org/0000-0003-3078-6465
}

Recepción: 25 de enero 2021

Revisado: 20 de febrero 2021

Aprobación: 30 de abril 2021

Publicación: 15 de mayo 2021 


\title{
RESUMEN
}

La investigación tuvo por objetivo analizar la práctica de la halterofilia a edades tempranas. Consecuencias en el crecimiento. Metodológicamente fue de tipo documental descriptivo. Entre los principales resultados, se tiene que es posible determinar que la practica de la Halterofilia a edades tempranas definitivamente es beneficioso para la salud y que este Deporte no acarrea consecuencias negativas sobre su crecimiento, así mismo, se puede concluir indicando que, actualmente el entrenamiento de la fuerza es considerado una actividad esencial para garantizar un adecuado rendimiento físico aplicado a cualquier deporte y que la mayor parte de autores sugieren la práctica de este Deporte, incluyendo entidades que velan por la seguridad y bien estar de los niños y adolescentes.

Descriptores: Deporte; medicina deportiva; efectos fisiológicos. (Palabras tomadas del Tesauro UNESCO).

\begin{abstract}
The research aimed to analyze the practice of weightlifting at an early age. Consequences on growth. Methodologically it was descriptive documentary type. Among the main results, it is possible to determine that the practice of Weightlifting at an early age is definitely beneficial for health and that this Sport does not have negative consequences on their growth, likewise, it can be concluded by indicating that, currently training Strength is considered an essential activity to guarantee adequate physical performance applied to any sport and that most authors suggest the practice of this Sport, including entities that ensure the safety and well-being of children and adolescents.
\end{abstract}

Descriptors: Sport; sports medicine; physiological effects. (Words taken from the UNESCO Thesaurus). 


\section{INTRODUCCIÓN}

La práctica de la halterofilia a edades tempranas es un tema que ha generado discusión y polémica en el campo de la actividad deportiva y salud, existen mitos y creencias sobre la influencia que ocasiona este deporte, principalmente en lo referente al crecimiento y desarrollo motor, por lo que es necesario realizar un estudio profundo de las consecuencias que se tiene cuando se desarrolla la fuerza a edades tempranas considerando que, estas pueden ser positivas o negativas.

Durante los últimos años han existido ciertos estudios que aprueban el desarrollo de la fuerza en niños, así como también autores que indican que puede influenciar de una manera negativa en el crecimiento de los deportistas, por ejemplo (DomínguezLa-Rosa \& Espeso-Gayte, 2003), sustentan que existen factores que impiden el desarrollo de la fuerza en deportistas prepúber, así como también, (Peña, et al., 2016) exteriorizan que "no existen pruebas científicas que demuestren que el entrenamiento de fuerza, correctamente supervisado y prescrito, pueda estar contraindicado en edades precoces" $\mathrm{p} 48$.

Por otra parte, se estima que del $70 \%$ al $90 \%$ de la estatura adulta está determinada genéticamente, en concordancia de los factores nutricionales y socioeconómicos (Rosenbloom, 2007). También la Organización Mundial de la Salud (OMS), hizo su publicación el 27 de abril del 2006 (Ginebra) donde indicaba que todos los niños del mundo tienen las mismas oportunidades de crecimiento, siempre y cuando reciban una atención óptima desde su nacimiento. Por tal motivo es interesante conocer y comprobar si la práctica de la Halterofilia tiene incidencia directa en estos dos factores. Según el diccionario de la (Real Academia Española, 2020), la Halterofilia es un deporte olímpico de Levantamiento de Pesas, se deriva de dos palabras haltera y filia; la haltera es una barra metálica con una bola o con discos en cada extremo y filia representa simpatía o afición por una determinada persona o cosa, es decir, significa afición por el levantamiento de pesas. La Federación Internacional de Halterofilia (International Weightlifting Federation), fundada en 1905 es el ente encargado de organizar y controlar el levantamiento de pesas a escala internacional y para el desarrollo de las competencias establece en su reglamento dos levantamientos: 
arrancada y dos tiempos; ambos levantamientos deben ser ejecutados con las dos manos y se permite un máximo de tres intentos en cada modalidad.

A pesar de que este Deporte se lo practica a nivel mundial, la mayoría de la población, incluyendo profesores de Educación Física y profesionales del Deporte, desconocen cuál es la edad óptima para empezar con el desarrollo de la fuerza o la iniciación deportiva en la halterofilia, ignoran si el uso de la fuerza está relacionado directamente con la salud, confort, sobre todo con el crecimiento del deportista. Además, en el país no está determinado la carga de entrenamiento y que programa se debe aplicar durante estas edades, así poder controlar la integridad física de los deportistas que se inician en esta disciplina deportiva, esto se debe a que la mayoría de entrenadores, son ex deportistas que se manejan empíricamente o como a ellos les enseñaron sin bases científicas que avalen sus procesos.

En otro sentido los docentes de Educación Física abordan este tema muy poco, ya que en esta área se busca el desarrollo de capacidades básicas y no específicamente el desarrollo de la fuerza, por otro lado, este deporte no es promocionado y abalizado por los padres de familia, por temor a que sus hijos pierdan la secuencia en su crecimiento.

En síntesis, durante mucho tiempo ha existido esta interrogante con respecto a la iniciación de la mejora de la fuerza en edades que comprenden entre los 11 y 14 años, por lo que se pretende realizar una revisión bibliográfica sistemática de carácter descriptivo exploratorio, analizar y comparar teóricamente la incidencia, ventajas y desventajas propuestas por diferentes autores en relación a la práctica de la Halterofilia en edades tempranas y el crecimiento y de esta manera proporcionar información adecuada, científicamente comprobada, para romper los miedos, temores y mitos que existen sobre este deporte llamado Halterofilia.

En razón de lo planteado, la investigación tiene por objetivo analizar 


\section{DESARROLLO}

Al realizar un estudio de las diferentes capacidades básicas que se desarrollan con la práctica de la Halterofilia, en edades que comprenden entre los 11 y 14 años de edad, el desarrollo de la fuerza constituye un componente extra, ya que, se han establecido mitos sobre esta práctica, mitos como por ejemplo que el deportista pierde la flexibilidad articular, el entrenamiento de la fuerza interfiere en su crecimiento, al entrenar con peso el deportista prepúber está expuesto fácilmente a lesiones deportivas, el niño sufre lesiones cardiacas, entre otras. Sin duda, fueron resultados y definiciones precoces publicados por algunos autores, sin embargo, se puede decir que la fuerza juega un papel muy importante como parte del individuo, ya sea como capacidad física básica, como dirección deportiva determinante o condicionante en el momento de la competición e incluso proporciona las garantías necesarias para que el deportista desarrolle sus acciones motrices de una manera secuencial y efectiva. (Siff \& Verkhoshansky, 2000).

La característica principal de la Halterofilia es el uso de la fuerza, según el diccionario de la (Real Academia Española, 2020), la fuerza es la capacidad para soportar un peso o resistir un empuje, en el ámbito deportivo y en las diferentes bases científicas se encuentra una variedad de conceptos y definiciones de varios autores que han aportado con su definición de fuerza, entre ellos (González \& Rivas, 2002), quienes definen la fuerza desde una perspectiva sistemática, sustentando que, la fuerza es la capacidad que posee el músculo para originar una elasticidad, durante su activación. Para (Verkhoshansky, 1999), la fuerza es el resultado de una activación muscular, la misma que presenta un proceso isócrono en todas las corrientes nerviosas del ser humano. (Knuttgen \& Kraemer, 1987) delimitan la fuerza como el mayor esfuerzo del músculo en un tiempo determinado, cuyo resultado de la presión que ejerce la persona contra el objeto produce la potencia y finalmente, (Vorobyev, 1978) la define como aquella tensión máxima que desarrollan los músculos y cuyo efecto es posible medir en gramos o kilogramos.

En base a cada uno de estos conceptos se puede establecer que la fuerza es generada por un estímulo que tiene la capacidad de cambiar el estado de reposo a un 
estado de movimiento en el músculo, todo este proceso se lleva a efecto gracias a que el músculo del ser humano está formado por fibras musculares que a su vez contienen miofibrillas, las partes encargadas de la contracción muscular al momento de realizar cualquier esfuerzo.

Por lo tanto, el sistema nervioso central realiza una regulación efectiva de la fuerza durante la ejecución de todos los movimientos corporales, brindándole al deportista la estabilización de los los niveles de fuerza conforme a cada movimiento o gesto técnico. (Siff \& Verkhoshansky, 2000) permitiendo que, el ser humano cumpla la mayor cantidad de actividades en el menor tiempo posible, a la vez que se produce una adecuada función celular y una apropiada producción de Adenosín Tri Fosfato (ATP), para su posterior utilización. En esta fuente de energía tiene origen el sistema de fosfágenos de alta energía, encargada de proporcionar al ser humano la capacidad o fuerza suficiente para cumplir determinadas actividades (Álvarez-Velázquez, et al. 2006).

Teniendo claro la definición de la fuerza y entendiendo que, es la capacidad física básica de mayor relevancia en la práctica de la Halterofilia, además que forma parte de manera notable en las acciones motoras del ser humano, ya que garantiza una eficaz ejecución de gestos técnicos e improvisados. (Siff \& Verkhoshansky, 2000), es necesario determinar la edad más apropiada para comenzar con su desarrollo y de esta manera establecer las vías a seguir para una práctica eficaz de la Halterofilia.

(Pastor, 2007), en la revista publicada por Journal of Human Sport and Exercise online, declara, que un gran número de autores que realizaron estudios sobre el momento oportuno para empezar con el desarrollo sistemático de la fuerza señalaban la última etapa de la adolescencia, (16 - 18 años), debido a que en esta etapa el cuerpo produce la mayor cantidad de hormonas androgénicas anabólicas (principalmente, la testosterona), sin embargo con el pasar de los años este tema genero mayor interés científico y al parecer los criterios han ido tomando otro contexto ya que la fuerza como el resto de factores motores (condicionales y coordinativos) alcanza su mayor desarrollo durante la fase sensible. 
Por otra parte, se conoce que el sistema nervioso central ejerce un minucioso control en cada una de las acciones técnicas específicas que el deportista emplea al realizar levantamientos de pesas o halteras, gracias a la innumerable cantidad de nervios que están distribuidos por todo el cuero (Siff \& Verkhoshansky, 2000). Inclusive, actualmente se estima que la preparación, iniciación y desarrollo de la fuerza a edades tempranas proporciona al deportista un adecuado desempeño físico, ayuda como coadyuvante en el desarrollo de otras capacidades físicas básicas y finalmente que facilita el rendimiento para cualquier disciplina deportiva.

\section{Consecuencias en el crecimiento.}

Haciendo un análisis solo de crecimiento la (Torres, 2002), indica que es la capacidad que tiene un organismo para realizar una síntesis celular, en cambio (Arce, 2015) menciona que, el crecimiento y el desarrollo del niño se sustenta en dos pilares fundamentales: el patrón genético, es decir el total de genes que los padres transfieren a los hijos y los factores ambientales que permiten que estos genes se expresen en el desarrollo del niño.

Por otra parte, el crecimiento físico en los niños se mide por los cambios en el tamaño y / o composición corporal, así como por el perfil físico (Ortega et al., 2008). Un dato importante también surgió en el Congreso Nacional de la Sociedad Española de Pediatría extra hospitalaria y atención primaria Santander junio 2017, donde se declara que cada ser humano que viene al mundo tiene su progreso de desarrollo y crecimiento ya establecido, también se indica que, mientras mas pronto empiece la etapa de la pubertad mayor será la estatura que alcance.

Aunque no exista información bibliográfica muy extensa a cerca de la influencia de la altitud sobre el crecimiento, en un estudio realizado en Lima se comprobó que, los niños que viven en elevadas altitudes presentan valores inferiores de crecimiento en comparación con otros niños que viven en lugares de menor altura (Cossio, et al., 2010). 
También es importante conocer cuál es la medida de crecimiento del ser humano en edades apegadas a la pubertad, entre algunos aportes importantes tenemos el de (Santiago, 2003) en la Revista Chilena de Nutrición, donde indica la diferencia entre el desarrollo de la estatura de un niño y el desarrollo de la estatura de una niña, esta etapa conocida también como el cambio de la infancia a la edad adulta está presente en el varón aproximadamente cuatro años y medio, donde se estima que en los dos años y medio crece de una manera muy acelerada mientras que este fenómeno cambia durante los próximos dos años donde es notable que su crecimiento se desarrolla de forma más lenta.

Durante todo este proceso el varón desarrolla su estatura mas o menos $25 \mathrm{~cm}$. Mientras que, en las niñas el proceso es de cuatro años, esta etapa se divide para que en los dos primeros años exista un crecimiento acelerado y en los dos años siguientes el crecimiento sea a una menor velocidad, creciendo aproximadamente $23 \mathrm{~cm}$. Tomando en cuenta estos valores de referencia, es importante analizar el desarrollo de la fuerza en estas edades y conocer un programa de entrenamiento ideal, para que el crecimiento del prepúber no se vea afectado a consecuencia de la práctica de la Halterofilia.

Relacionando el crecimiento y la fuerza a edades tempranas es necesario también establecer cuáles son los riesgos o beneficios que se obtiene y cuáles son las consecuencias al empezar con la práctica de la halterofilia a edades tempranas, afirmando así o descartando las creencias y mitos sobre la práctica de la Halterofilia y sus efectos negativos en el crecimiento. Con respecto a este tema, (Pastor, 2007), expone que, en el año de 1985 la Sociedad Americana de Ortopedia para la Medicina del Deporte, la Asociación Americana de Pediatría, el Consejo Americano de Medicina del Deporte, la Asociación Nacional de Fuerza y Acondicionamiento, el Comité Olímpico Americano, la Sociedad de Pediatría y Ortopedia, exponían que el desarrollar la fuerza a edades tempranas causa efectos favorables sobre el organismo de los adolescentes. 
Considerando como base los diferentes aportes de las entidades que dedican sus esfuerzos y recursos a la salud, seguridad y bienestar de los niños y adolescentes, se puede mencionar algunos beneficios, entre ellos tenemos la disminución de lesiones y patologías deportivas, perfeccionamiento de las diferentes habilidades y técnicas, progreso efectivo de la fuerza muscular y buen desempeño en el aparato locomotor (mejorar otras capacidades de tipo deportivo), mejora de la salud ósea (aumenta la densidad y contenido mineral óseo), mejoras de la composición corporal y ayuda en el proceso de la sensibilidad a la insulina (Peña, et al., 2016).

Desglosando lo manifestado por (Peña, et al, 2016), el sistema músculo esquelético está constituido por la unión de los huesos, las articulaciones y los músculos, proporcionando al ser humano movilidad, sostén y con el desarrollo de la fuerza a edades tempranas se obtendrá un componente adicional para que el cuerpo del deportista se adapte a un mayor esfuerzo físico y consecuentemente a un mayor rendimiento deportivo; en lo referente a la ayuda que brinda el desarrollo de la fuerza sobre la sensibilidad a la insulina, se entiende que brindará una eficaz acción de la insulina en el cuerpo, provocando que la glucosa que penetra en el torrente sanguíneo genere la energía necesaria y adecuada para el normal funcionamiento del cuerpo.

Un aporte similar es el que hace (Faigenbaum, et al. 2010) en el artículo denominado "entrenamiento de resistencia pediátrico", donde manifiesta que los hallazgos recientes indican que el entrenamiento de resistencia a la fuerza puede ofrecer beneficios únicos para niños y adolescentes cuando la práctica se prescribe y es supervisada. Además de optimizar la fuerza ejercida por el músculo y mejorar el rendimiento de las diferentes acciones motrices, la participación regular en un entrenamiento de resistencia pediátrico puede facilitar el control del peso, fortalecer los huesos y aumentar la resistencia de un atleta joven a las lesiones relacionadas con el deporte.

De igual manera (Domínguez, et al., 2016) declaran que el efecto de trabajo contra resistencias o "Resistance Training" ayuda a prevenir algunas enfermedades como por ejemplo la obesidad, hipertensión arterial, diabetes mellitus, esclerosis múltiple, 
entre otros, como también puede servir de coadyuvante para la curación de algunas patologías.

En la halterofilia se ejecutan movimientos de flexión y extensión articular y generalmente se desarrolla un plan de entrenamiento general a la fuerza por lo que es imprescindible mencionar y conocer que pasa con el cartílago del crecimiento. La fisis o cartílago de crecimiento es una zona ubicada en la metatasis de los huesos, que produce el crecimiento longitudinal de los huesos largos (Shapiro \& Forriol, 2005). El cartílago sano ayuda a moverse, al permitir que los huesos se deslicen por encima de los otros. En un estudio realizado en Colombia denominado "Cartílago de crecimiento y altura de la epífisis proximal tibial en halterófilos" cuyos autores fueron (Quiroz-Meneses, et al. 2007), realizaron un estudio con deportistas federados de 14 años, quienes practicaban de manera ordenada un programa de levantamiento de pesas, cuyo objetivo de estudio fue realizar una comparación con atletas que practicaran otra disciplina deportiva y determinar que sucede con el grosor del cartílago de crecimiento y la altura de la epífisis proximal tibial, se obtuvo como resultado que no existía alteraciones con la densidad del cartílago de crecimiento, al igual que se pudo determinar que no hay cambios con la elevación de la epífisis proximal tibial en comparación con deportistas que practicaban otros deportes. Esto sustenta un dato muy importante ya que es un indicador que no se ve alterado la etapa de crecimiento, cuando de desarrolla la fuerza a edades tempranas.

En complemento, (Navarro, 2007) indica los beneficios que ocasiona el entrenamiento de la fuerza en niños y adolescentes, mencionando que brinda ayuda a nivel de todo el aparato locomotor, siempre y cuando los ejercicios de resistencia a la fuerza cumplan con una planificación y guía o supervisión de un profesional, otro beneficio es el psicosocial, donde se manifiesta que el desarrollo de la fuerza influye directamente sobre los niveles de ansiedad, estrés y depresión, reduciendo los mismos en un gran porcentaje, también se indica que los prepúber que practican el entrenamiento de resistencia a la fuerza están menos propensos a padecer de obesidad o sobrepeso, ya que existe un mejor desempeño fisiológico, mayor consumo 
de ácidos grasos y consecuentemente va existir la reducción del tejido adiposo a nivel general.

Sin embargo, se menciona también que puede existir ciertos riesgos: cuando existe la ausencia de supervisión calificada, si los elementos de entrenamiento se utilizan de manera inadecuada, si la ejecución de la técnica es defectuosa, también se da cuando existe errores en la programación del volumen, intensidad y la densidad de la carga, olvidando el principio del aumento gradual de las cargas de trabajo. Todo esto está relacionado con los principios que se debe respetar en el entrenamiento de la fuerza tales como: principio de Progresión. (adaptación), principio de la regularidad (con una frecuencia semanal de dos a tres veces por semana), principio de sobrecarga (disminuye el fenómeno de huella), principio de creatividad (optimizar el interés), principio de diversión (disfrutar del entrenamiento), principio de socialización (interactuar) y el principio de supervisión (profesional capacitado).

Adicionalmente (Barbieri \& Zaccagni, 2013) afirman que, el entrenamiento de fuerza es una práctica relativamente segura y saludable para los niños, siempre y cuando se conceda autorización médica y se realice los entrenamientos con el calentamiento, estiramiento y manejo de cargas adecuadas.

Por otra parte, no debemos confundir el amor al levantamiento de pesas con la Dismorfia muscular, descrita como, una alteración de la imagen corporal, en esta alteración los deportistas presentan una exagerada preocupación por alcanzar un cuerpo lleno de músculos y de aspecto físico perfecto, desarrollando conductas desadaptativas. (Pope, et al., 1997), ventajosamente no existen registros de prepúberes que hayan padecido esta enfermedad.

Finalmente se ha comprobado que, la planificación deportiva adaptada al desarrollo de la fuerza y planificada con ejercicios de resistencia a la fuerza, ayuda a disminuir el tejido adiposo e incrementa las habilidades para la ejecución del salto y del lanzamiento en niños y jóvenes. (Casas, et al., 2018). 


\section{Objetivos para el desarrollo de la fuerza en niños y niñas}

El deporte en general pretende educar aptitudes de coordinación y mejora de la técnica, por lo que es imprescindible establecer cuáles son los objetivos que se desea alcanzar con el entrenamiento de la fuerza en las edades prepúber, (Vasconcelos, 2005), presenta de manera muy concreta los objetivos que se deben buscar, estos son, desarrollar en el deportista el fenómeno de huella, es decir adquirir los mejores gestos técnicos, generar en el deportista buenos hábitos de vida, con el fin de evitar lesiones, fomentar una buena vinculación del cuerpo con el espacio exterior y finalmente el desarrollo tanto de la fuerza explosiva como de la fuerza resistencia (López-Gallego, et al. 2015).

(García, et al., 2010), sugiere tener presente la regla general de la frecuencia semanal de entrenamiento, indicando que, se debe considerar máximo dos sesiones por semana para los deportistas que están iniciándose en el desarrollo de la fuerza, además afirma que la fuerza muscular puede ser transformada hasta un $67 \%$ en los niños.

En este sentido, (Martin, et al. 2004), defienden su posición indicando que el objetivo primordial en edades escolares es preparar al deportista a largo plazo, mas no buscar en Él los mejores resultados deportivos. Inclusive (Faigenbaum \& Myer, 2006), citado por (García, et al. 2010) ha considerado idóneo los ejercicios pliométricos para el desarrollo de la fuerza explosiva en niños.

\section{Planificación Dietético Nutricional}

A todo lo mencionado sobre el crecimiento y el desarrollo de la fuerza a edades tempranas se puede acotar también un tema muy importante que es la alimentación, ya que el deportista, entrenadores e incluso la familia debe conocer cuál es la alimentación adecuada para que se produzca un correcto gasto energético en el deportista; existe varias opciones sin embargo, la que mas prevalece, es el consumo de alimentos ricos en proteína, tales como el bacalao, pechuga de pollo o pavo, atún lentejas, entre otros, considerando que el consumo de estos alimentos ayuda al 
deportista a reparar al músculo, a mas de contribuir para su crecimiento y normal desarrollo. (Aguirre, et al., 2010).

\section{Orientaciones metodológicas para el entrenamiento de la Halterofilia en niños.}

Considerando la importancia de este deporte y al saber que somos responsables de la vida de personas que empiezan a descubrir nuevas experiencias, entre ellas descubrir el mundo deportivo, es necesario conocer y estructurar un programa adecuado de entrenamiento para el inicio de la fuerza. Para cumplir con un programa de entrenamiento adecuado es necesario conocer las bases científicas del entrenamiento deportivo y reconocer la evolución y cambios de los diferentes métodos de entrenamiento.

En primer lugar, debemos tener claro el proceso de periodización, este proceso nos permite establecer un orden determinado para cada uno de los ejercicios, además permite el aumento del potencial motor y consecuentemente se podrá alcanzar la mejor forma deportiva (Bompa y Buzzichelli, 2015). Considerando el tema de la metodología (Navarro, 2007), sugiere dos datos importantes, el primero es el dominio de la técnica y el segundo donde indica que los implementos deportivos que se utilizan durante las sesiones de entrenamiento deben ajustarse a las características somáticas y anatómicas de los deportistas adolescentes.

Por otra parte, (Peña, et al., 2016) recomienda iniciar cada sesión de entrenamiento con ejercicios que permitan la activación de todo el sistema corporal, evadir gestos deportivos con altas cargas de intensidad para la columna vertebral, tener como alternativa ejercicios que aporten a la mejora de la coordinación y el equilibrio, sugiere aplicar el principio de la variación ondulatorio de las cargas, es decir, aplicar en el plan de entrenamiento un incremento gradual de la carga volumen e intensidad y finalmente establece que se deben utilizar sistemas de entrenamiento que favorezcan a la motivación, diversión y concentración del niño.

Como entrenadores o Docentes de Educación Física es necesario tener claro el objetivo que se desea alcanzar en cada una de las clases o sesiones de entrenamiento y planificar las actividades controlando los momentos de descanso entre el volumen y 
la intensidad del trabajo, en lo referente a este tema (Faigenbaum \& Myer, 2010), manifiesta que un intervalo de descanso aproximadamente de un minuto puede ser suficiente en niños y adolescentes cuando se realiza un protocolo de ejercicios de resistencia a la fuerza. Finalmente, este mismo autor indica que el entrenamiento de resistencia con pesas libres, balones medicinales y el propio peso corporal puede ser particularmente beneficioso para los atletas jóvenes que necesitan mejorar el rendimiento de las habilidades motoras, el equilibrio, la fuerza central y la potencia muscular como parte de un programa de entrenamiento integrado.

\section{APORTES}

Se ha realizado un estudio sistemático descriptivo, de revisión bibliográfica, pues se busca detallar de forma crítica y exhaustiva estudios previamente publicados sobre la "Práctica de la Halterofilia a edades tempranas: consecuencias en el crecimiento". Para la búsqueda de información se utilizaron fuentes primarias como son las revistas científicas con publicaciones originales con información reciente sobre el tema (Erazo-Álvarez \& Narváez-Zurita, 2020). También se utilizaron fuentes secundarias como son las bases de datos electrónicas: Scielo, Google Académico, Dialnet, Pubmed, Scopus Elsevier, Redalyc.

Para obtener una información sólida y adecuada se utilizó algunos criterios de selección como: artículos que tengan mayor impacto (Q1, Q2, Q3, Q4), en las diferentes bases de datos, se utilizó una búsqueda con filtro por año de publicación del 2010 al 2020, artículos relacionados al desarrollo de la fuerza a edades tempranas, además se utilizó como medio y estrategia de búsqueda las palabras claves como fuerza, crecimiento, halterofilia. Artículos en inglés y en español (Parra-Zhizhingo, et al. 2020).

Como resultado de le Investigación Bibliográfica, sobre la Práctica de la Halterofilia a edades tempranas y sus consecuencias en el crecimiento, es posible concluir indicando que la práctica de la halterofilia a edades tempranas ha presentado una amplia evolución durante las últimas dos décadas. Este estudio se realizó considerando que la característica principal para la práctica de la Halterofilia es el uso 
de la fuerza, tomando como referencia este dato se puede indicar que entidades como la Sociedad Americana de Ortopedia para la Medicina del Deporte.

La Asociación Americana de Pediatría, entre otras, declaran que el desarrollo de la fuerza en edades tempranas puede ser sano y beneficioso para los más jóvenes, además la mayoría de autores indican que la práctica de la fuerza a edades tempranas puede brindar múltiples beneficios para los niños y adolescentes, incluyendo la reducción de la adiposidad corporal, evitar la obesidad infantil y la ayuda en el desempeño motriz (Yépez-Ormaza, et al. 2020).

Se puede considerar que, actualmente el entrenamiento de la fuerza es considerado una actividad esencial para garantizar un adecuado rendimiento físico aplicado a cualquier deporte. También se debe recalcar que la practica de la halterofilia a edades tempranas no acarrea consecuencias negativas sobre el crecimiento del prepúber y específicamente no existe afección en el cartílago de crecimiento, por lo que se descarta la teoría de que los halterófilos quedan de baja estatura. Todos estos beneficios se alcanzan cuando se ejecuta un buen programa de entrenamiento, bajo supervisión profesional, la ayuda de un médico y la ayuda en casa (correcta alimentación).

Finalmente se invita a todas las personas que de una u otra manera se encuentran vinculados en el campo educativo y deportivo, a seguir investigando este tema para así evitar cometer errores sobre todo a edades tempranas y así masificar la práctica de la halterofilia con miras a alcanzar el alto rendimiento en la arena competitiva nacional e internacional.

\section{FINANCIAMIENTO}

No monetario.

\section{AGRADECIMIENTO}

A la Universidad Católica de Cuenca y la Jefatura de Posgrados por apoyar el desarrollo de la investigación 


\section{REFERENCIAS CONSULTADAS}

Aguirre M.L, Castillo C, \& Le Roy C. (2010). Desafíos Emergentes en la Nutrición del Adolescente [Emergent Challenges in Adolescent Nutrition]. Revista chilena de pediatría, 81(6), 488-497. https://dx.doi.org/10.4067/S0370$\underline{41062010000600002}$

Álvarez-Velázquez, I, Álvarez-Velázquez, F., Álvarez-Barreras, F, \& Mena-Ramos, R. (2006). El proceso de asimilación de la fuerza en el músculo del ser humano [The process of assimilation of force in the muscle of the human being]. $R a$ Ximhai, 2(2),533-548.

Arce, M. (2015). Crecimiento y desarrollo infantil temprano [Early Childhood Growth and Development]. Revista Peruana de Medicina Experimental y Salud Pública, 32(3), 574-578. https://doi.org/10.17843/rpmesp.2015.323.1694

Barbieri, D., \& Zaccagni, L. (2013). Strength training for children and adolescents: benefits and risks. Collegium antropologicum, 37 Suppl 2, 219-225.

Bompa T, \& Buzzichelli C. (2015). Periodization Training for Sports Third Edition. Human Kinetics. Estados Unidos. Tercera edición.

Casas, Á, Naclerio, F., Dopico-Calvo, X., \& García, C. (2018). Efectos del entrenamiento de la aptitud muscular sobre la adiposidad corporal y el desempeño motriz en niños y jóvenes: un meta-análisis [Effects of muscle fitness training on body adiposity and motor performance in children and youth: a meta-analysis]. Educación Física Y Ciencia, 20(2), e046. https://doi.org/10.24215/23142561e046

Cossio M., Arruda M., Núñez V., \& Lancho J. (2010). Efectos de la altitud sobre el crecimiento físico en niños y adolescentes [Effects of altitude on physical growth in children and adolescents]. Rev Andal Med Deporte, 4(2):71-76.

Domínguez, R., Garnacho-Castaño, M, \& Maté-Muñoz, J. (2016). Efectos del entrenamiento contra resistencias o resistance training en diversas patologías [Effects of resistance training in various pathologies]. Nutrición Hospitalaria, 33(3),719-733.

Domínguez-La-Rosa, P. \& Espeso-Gayte, E. (2003). Bases fisiológicas del entrenamiento de la fuerza con niños y adolescentes [Physiological bases of strength training with children and adolescents]. Revista Internacional de Medicina y Ciencias de la Actividad Física y el Deporte, 3 (9), 61-68. 
Erazo-Álvarez, J., \& Narváez-Zurita, C. (2020). Medición y gestión del capital intelectual en la industria del cuero - calzado en Ecuador [Measurement and management of intellectual capital in the leather industry - footwear in Ecuador]. Revista Arbitrada Interdisciplinaria Koinonía, 5(9), 437-467. http://dx.doi.org/10.35381/r.k.v5i9.662

Faigenbaum, A. D., \& Myer, G. D. (2010). Pediatric resistance training: benefits, concerns, and program design considerations. Current sports medicine reports, 9(3), 161-168. https://doi.org/10.1249/JSR.0b013e3181de1214

García O; Serrano V; Martínez I; Cancela J. (2010). La fuerza: ¿una capacidad al servicio del proceso de enseñanza-aprendizaje de las habilidades motoras básicas y las habilidades deportivas específicas [Strength: a capacity at the service of the teaching-learning process of basic motor skills and specific sports skills]. Revista de Investigación en Educación, (8), 108-116.

González J., \& Ribas J. (2002). Bases de la programación del entrenamiento de fuerza [Basis of strength training programming]. Primera Edición. Editorial INDE Publicaciones. Barcelona - España.

Knuttgen H. \& Kraemer. (1987). Terminology and Measurement in Exercise Perfomance. Journal of Applied Sport Science Research, 1(1), 1-10.

López-Gallego, F., Lara-Sánchez, A., Espejo-Vacas, N, \& Cachón-Zagalaz, J. (2015). Evaluación de la fuerza explosiva de extensión de las extremidades inferiores en escolares [Evaluation of the explosive force of extension of the lower extremities in schoolchildren]. Apunts Educación Física y Deportes, (122),4451.

Martin D; Nicolaus J; Ostrowski J; Rost K. (2004). Metodología general del entrenamiento infantil y juvenil [General methodology of child and youth training]. Primera edición. Editorial Paidotribo. Barcelona

Muzzo B, S. (2003). Crecimiento normal y patológico del niño y del adolescente [Normal and abnormal growth of the child and the adolescent]. Revista chilena de nutrición, 30(2), 92-100. https://dx.doi.org/10.4067/S0717$\underline{75182003000200003}$

Organización Mundial de la Salud. (2006). La OMS difunde un nuevo patrón de crecimiento infantil [WHO spreads a new pattern of child growth]. https://n9.cl/1hy6b 
Ortega, F. B., Ruiz, J. R., Castillo, M. J., \& Sjöström, M. (2008). Physical fitness in childhood and adolescence: a powerful marker of health. International journal of obesity (2005), 32(1), 1-11. https://doi.org/10.1038/sj.ijo.0803774

Parra-Zhizhingo, Y., García-Herrera, D., Ávila-Mediavilla, C., \& Erazo-Álvarez, J. (2020). Plataformas Virtuales: retos y perspectivas a partir de Docentes [Virtual Platforms: challenges and perspectives from Teachers]. Revista Arbitrada Interdisciplinaria Koinonía, 233-249. doi:http://dx.doi.org/10.35381/r.k.v5i5.1041

Pastor F. J. (2007). El entrenamiento de la fuerza en niños y jóvenes. aplicación al rendimiento deportivo [Strength training in children and youth. application to sports performance]. Journal of Human Sport and Exercise, II(1),1-9.

Peña G., Heredia J.R, Lloret, C., Martín M; Da Silva M.E. (2016). Iniciación al entrenamiento de fuerza en edades tempranas. Revista Andaluza de Medicina del Deporte, (9), 1. https://www.redalyc.org/pdf/3233/323344442009.pdf

Pope, H. G., Jr, Gruber, A. J., Choi, P., Olivardia, R., \& Phillips, K. A. (1997). Muscle dysmorphia. An underrecognized form of body dysmorphic disorder. Psychosomatics, 38(6), 548-557. https://doi.org/10.1016/S0033$\underline{3182(97) 71400-2}$

Quiroz-Meneses, L.A., \& Celis Osorio, R., \& Granados, A. (2007). Cartílago de crecimiento y altura de la epífisis proximal tibial en halterófilos [Growth plate and height of the proximal tibial epiphysis in weightlifters]. Revista Internacional de Medicina y Ciencias de la Actividad Física y del Deporte / International Journal of Medicine and Science of Physical Activity and Sport, 7(27),185-202.

Real Academia Española (2020). Definición de fuerza [Definition of force]. https://www.rae.es/drae2001/fuerza

Real Academia Española (2020). Halterofilia [Weightlifting]. https://www.rae.es/drae2001/halterofilia

Rosenbloom A, L. (2007). Fisiología del crecimiento [Growth physiology]. Ann Nestlé [Esp] (65), 99-110. https://doi.org/10.1159/000151261

Shapiro. F., \& Forriol F. (2005). El cartílago de crecimiento: biología y biomecánica del desarrollo [Growth cartilage: developmental biology and biomechanics]. Revista Española de Cirugía Ortopédica y Traumatología, 49(1), 55-67.

Siff M., \& Verkhoshansky Y. (2000). Super entrenamiento [Super workout]. Primera Edición. Editorial Paidotribo. España. 
Torres, SAR. (2002). Crecimiento y desarrollo [Growth and development]. Rev Mex Med Fis Rehab, 14(2-4), 54-57.

Vasconcelos A. (2005). La fuerza. Entrenamiento para jóvenes Guía teórico-práctica [The force. Training for young people Theoretical-practical guide]. Editorial Paidotribo. España.

Verkhoshansky Y. (1999). Todo sobre el método pliométrico. Medios y métodos para el entrenamiento y la mejora de la fuerza explosiva [All about the plyometric method. Means and methods for training and improving explosive strength]. Segunda Edición. Editorial Paidotribo. España.

Vorobyev, A.N. (1978). A textbook on weightlifting. Publicado por la International Weighlifting Federation. Budapest. Recuperado de https://n9.cl/ctg7i

Yépez-Ormaza, P., García-Herrera, D., Cárdenas-Cordero, N., \& Erazo Álvarez, J. (2020). Plataformas digitales: Mundo primario como estrategia para el desarrollo del lenguaje en niños [Digital platforms: Primary world as a strategy for language development in children]. Revista Arbitrada Interdisciplinaria Koinonía, 5(5), 358-376. http://dx.doi.org/10.35381/r.k.v5i5.1048 